\title{
Growth kinetics of small renal masses: A prospective analysis from the Renal Cell Carcinoma Consortium of Canada
}

\author{
Michael Organ, MD; ${ }^{*}$ Michael Jewett, MD, FRCSC; ${ }^{\dagger}$ Joan Basiuk, RN; Christopher Morash, MD, FRCSC; \\ Stephen Pautler, MD, FRCSC,; D. Robert Siemens, MD, FRCSC;, Simon Tanguay, MD, FRCSC,F \\ Martin Gleave, MD, FRCSC, FACS,; Darrell Drachenberg, MD, FRCSC, ${ }^{; b}$ Raymond Chow; ${ }^{c}$ \\ Joseph Chin, MD, FRCSC; $;$ Andrew Evans, MD, PhD, FRCPC;, Neil Fleshner, MD, FRCSC; ${ }^{+}$ \\ Brenda Gallie, MD, FRCPC; ${ }^{;}$Masoom Haider, MD, FRCPC, J John Kachura, MD, FRCPC,f \\ Antonio Finelli, MD, FRCSC; ${ }^{\dagger}$ Ricardo A. Rendon, MD, FRCSC
}

*Faculty of Medicine, Department of Urology, Dalhousie University, Halifax, NS; 'Department of Surgery, Division of Urology, University of Toronto, Toronto, ON; Kidney Cancer Research Network of Canada, Toronto, ON; §Department of Surgery, Division of Urology, University of Ottawa, Ottawa, ON; *Division of Urology, Schulich School of Medicine and Dentistry, Western University, London, ON; ¿Department of Urology, Queen's University, Kingston, ON; EDivision of Urology, McGill University, Montreal, QC; 'Department of Urologic Sciences, University of British Columbia, Vancouver, BC; bFaculty of Medicine, Division of Urology, University of Manitoba, Winnipeg, MB; 'Department of Health Informatics, Princess Margaret Hospital, Toronto, ON; 'Department of Pathology and Laboratory, Faculty of Medicine, University of Toronto, Toronto, ON; e Department of Ophthalmology and Vision Sciences, University of Toronto, Toronto, ON; 'Department of Medical Imaging, University of Toronto, Toronto, ON

See related article on page 28 .

Cite as: Can Urol Assoc J 2014;8(1-2):24-7. htrp://dx.doi.org/10.5489/cuaj.1483 Published online February 10, 2014.

\section{Abstract}

Introduction: Most small renal masses (SRMs) are diagnosed incidentally and have a low malignant potential. As more elderly patients and infirm patients are diagnosed with SRMs, there is an increased interest in active surveillance (AS) with delayed intervention. Patient and tumour characteristics relating to aggressive disease have not been well-studied. The objective was to determine predictors of growth of SRMs treated with AS.

Methods: A multicentre prospective phase 2 clinical trial was conducted on 207 SRMs in 169 patients in 8 institutions in Canada from 2004 to 2009; in these patients treatment was delayed until disease progression. Patient and tumour characteristics were evaluated to determine predictors of growth of SRMs by measuring rates of change in growth (on imaging) over time. All patients underwent AS for presumed renal cell carcinoma (RCC) based on diagnostic imaging. We used the following factors to develop a predictive model of tumour growth with binary recursive partitioning analysis: patient characteristics (age, symptoms at diagnosis) and tumour characteristics (consistency [solid vs. cystic] and maximum diameter at diagnosis.

Results: With a median follow-up of 603 days, 169 patients (with 207 SRMs) were followed prospectively. Age, symptoms at diagnosis, tumour consistency and maximum diameter of the renal mass were not predictors of growth. This cohort was limited by lack of availability of patient and tumour characteristics, such as sex, degree of endophytic component and tumour location.

Conclusion: Slow growth rates and the low malignant potential of SRMs have led to AS as a treatment option in the elderly and infirm population. In a large prospective cohort, we have shown that age, symptoms, tumour consistency and maximum diameter of the mass at diagnosis are not predictors of growth of T1a lesions. More knowledge on predictors of growth of SRMs is needed.

\section{Introduction}

In 2013, renal cell carcinoma (RCC) was the third most commonly diagnosed genitourinary malignancy. ${ }^{1}$ As imaging techniques have advanced in recent years, there has been a trend towards renal tumour downward stage migration and smaller size at diagnosis, such that T1 tumours now account for most incidentally detected tumours. ${ }^{2}$ Early RCC is now more likely to be detected when patients are asymptomatic at diagnosis. ${ }^{3}$ Most of these small renal masses (SRMs) have a lower malignant potential and are defined as predominantly solid enhancing tumours $\leq 4 \mathrm{~cm}$ in maximal diameter. ${ }^{4}$ Traditionally, the treatment of these lesions has been total nephrectomy, but concerns about predisposing these patients to chronic renal failure ${ }^{5}$ and subsequently increased cardiac risk and shortened overall survival ${ }^{6}$ has led to nephron-sparing treatments. The standard of care for SRMs has changed; clinical guidelines now recommend partial nephrectomy for most T1 renal tumours; ${ }^{4}$ however, there is an evolving body of literature that suggests active surveillance (AS) of SRMs may be appropriate in the elderly and infirm population. ${ }^{7}$

Many patients in whom SRMs are detected are elderly and potentially have significant comorbidities. Half are over 65 years old when the SRM is detected, ${ }^{3}$ with the greatest increase in detection in patients between 70 and $90 .^{8}$ Previous retrospective studies have revealed that the growth 
rate for SRMs is slow, with very few progressing to metastatic disease. ${ }^{9}$ Up to $46 \%$ of those masses are benign. ${ }^{10}$ Considering that many elderly have a high-risk of perioperative morbidity and mortality, in those unfit for surgery, AS with delayed treatment until progression has been widely practiced. . $^{411,12}$

Patient and tumour characteristics related to more aggressive behaviour has not been well-studied. To develop algorithms and treatment plans for patients with SRMs, the natural history of these masses needs to be better understood as we currently do not have a good understanding about which lesions are safe to observe and which need immediate intervention. Tumour size is a poor predictor of benign or malignant lesions. ${ }^{13}$ Radiographic techniques do not accurately distinguish between benign versus malignant masses and renal biopsy is not sufficient to determine the histology of all SRMs. ${ }^{14}$ No correlation has been documented between tumour growth and age, presenting symptoms, solid/cystic consistency or tumour multifocality. $7,9,15,16$ We have published a series of 84 masses with the longest prospective follow-up to date (3 years); we found that tumour size is a predictor of the growth rate, with masses $>2.45 \mathrm{~cm}$ growing faster than those $<2.45 \mathrm{~cm}$. Others have reported conflicting results; smaller tumours actually grow faster than larger ones. ${ }^{16}$ The long-term natural history of SRMs remains unknown. We assess the predictors of growth rate from a large prospective phase 2 trial of patients followed at 8 institutions across Canada.

\section{Methods}

This institutional ethics board approved protocol included 207 renal masses that were prospectively followed in 169 patients; these patients were enrolled in a phase 2 clinical trial of AS with treatment delayed until progression. Eight centres across Canada (Renal Cell Carcinoma Canadian Consortium) collaborated to enroll and follow patients. The inclusion criteria included $\mathrm{T} 1 \mathrm{aNOMO}$ renal masses on imaging in patients who were considered unfit for surgery by their physician due to advanced age, comorbidity or refusal of other treatments. The exclusion criteria included a life expectancy of $<2$ years, a diagnosis of a SRM $>12$ months before accrual, patients who were on concurrent systemic therapy for other malignancies, or had a known familial RCC.

Patient and tumour characteristics were collected. Patient age was recorded in years. Patients were deemed symptomatic if they had hematuria or pain thought to be caused by a renal mass. Maximum diameter of the mass was determined in centimeters for each tumour at every image point. Tumours were determined to be cystic or solid based on imaging. Cystic renal masses were those with a visible area of fluid and displaying features suggestive of malignancy according to the Bosniak classification. ${ }^{17}$ Serial imaging was performed with computed tomography, magnetic resonance imaging, or ultrasound depending on patient body habitus and/or physician preference at about 3 and 6 months from diagnosis and every 6 months thereafter.

\section{Statistical analysis}

Tumour growth rates were calculated as an increase in maximum diameter over time stated as $\mathrm{cm} /$ year. Recursive partitioning analysis (RPA) for repeated measured outcomes was used to develop a predictive model of tumour growth ${ }^{18,19}$ using maximum diameter at diagnosis, tumour location, consistency (solid vs. cystic), patient age, and symptoms at diagnosis as the potentially predictive variables. The RPA algorithm automatically ranked all the dichotomous splits of the data for each of the potential prognostic factors (covariates) included in the model; a goodness-of-split measure was selected as the optimal overall split at that node. The process was applied recursively to nodes until further splitting achieved negligible improvement in the model, resulting in a simple model presented as a sequence of decision rules.

\section{Results}

With a median follow-up of 603 days, (1.7 years; range: 0.25-8 years), 169 patients with 207 renal masses were enrolled in the study from 2004 to 2009. The mean age was 72.5 years (range: $52-91$ ). Symptoms were present in $8.4 \%$ of patients at diagnosis, while $91.6 \%$ were asymptomatic. Solid masses were present in $85.8 \%$ of patients, while $14.2 \%$ were cystic. Histological outcomes, local progression and metastasis data have been previously published.

There was a median of 5 radiographic studies per mass (range: 2-14). The median size of the masses at diagnosis was $2.15 \mathrm{~cm}$ (standard deviation: 0.79). The median growth rate of the entire cohort was $0.12 \mathrm{~cm} /$ year (standard deviation: 0.016) (Table 1).

The automatic variable selection feature of the repeated RPA algorithm did not identify any variables as predictors of growth. Age, symptoms at diagnosis, tumour consistency, and maximum diameter at diagnosis were not predictors of growth.

\section{Discussion}

The standard of care for the treatment of organ-confined renal masses for most patients is partial or radical nephrectomy. Retrospective series demonstrating slow growth rates and a low malignant potential have led to an increased interest in AS of SRMs. Despite a recent increase in AS for elderly patients with SRMs, the natural history of these masses is not 
Organ et al.

\begin{tabular}{lcc}
\hline \multicolumn{3}{l}{ Table 1. Descriptive statistics of cohort } \\
\hline \multicolumn{1}{l}{ Patient characteristics } & & \\
\hline Follow-up (median) & 1.7 years & $\begin{array}{c}\text { Range }(0.25-8 \\
\text { years) }\end{array}$ \\
No. patients & 169 & - \\
No. masses & 207 & - \\
Imaging studies/mass (median) & 5 & Range (2-14) \\
Age (mean) & 72.5 & Range (52-91) \\
Symptomatic (\%) & 8.4 & - \\
Asymptomatic (\%) & 91.6 & - \\
Tumour characteristics & & \\
Size (median) & $2.15 \mathrm{~cm}$ & $\mathrm{SD}(0.79)$ \\
Growth rate (median) & $0.12 \mathrm{~cm} / \mathrm{year}$ & $\mathrm{SD}(0.016)$ \\
Solid & $85.8 \%$ & - \\
Cystic & $14.2 \%$ & - \\
\hline
\end{tabular}

well-known. As patients continue to survive into their elderly years and as more incidental tumours are detected, there is an increasing need to learn about the growth characteristics of these lesions.

It has been demonstrated that larger tumours have more aggressive features, ${ }^{20}$ with tumours smaller than $3 \mathrm{~cm}$ less likely to metastasize. ${ }^{21}$ In a pooled statistical analysis of multiple retrospective AS series, only lesions that displayed growth were capable of developing metastases. ${ }^{22}$ Despite the presumed importance of size and growth rate of SRMs, there is limited data on the growth kinetics of tumours after diagnosis. Predictors of growth have been controversial; only 1 study suggests that smaller masses grow faster ${ }^{16}$ and another study suggests smaller masses grow slowly. ${ }^{9}$

In this large prospective multi-institutional cohort, patient age, symptoms at diagnosis and tumour consistency were not predictive of tumour growth. This is consistent with our smaller prospective study, as well as with multiple retrospective publications. ${ }^{9,15,16}$ Additionally, in this study, maximum tumour diameter at diagnosis was not a predictor of growth. This new data contradict a previous prospective, but smaller, cohort of different patients from our institution. This previous study suggested that a maximum diameter of $>2.45 \mathrm{~cm}$ predicted an increased growth rate compared to SRMs smaller in size. This contradictory finding can be substantiated by the fact that the current cohort study is much larger (207 masses vs. 84 masses) and has a shorter median follow-up time (1.7 years). Another reason for the discrepancy could be that the current cohort includes only cT1a lesions, while Mason and colleagues included cT1a with some cT1b lesions (median maximum diameter: 2.15 vs. $2.30 \mathrm{~cm}) .{ }^{9}$ It is possible that larger renal lesions may display different growth characteristics than smaller ones. Finally, the cohort from Mason and colleagues reported a larger growth rate of $0.24 \mathrm{~cm} /$ year versus $0.12 \mathrm{~cm} /$ year. It is hypothesized that it would be easier to identify prognostic factors of tumour growth when renal masses display faster growth rather than a near flat growth rate.

To our knowledge, this is the largest published prospective study looking at predictors of growth rate in AS for renal masses. However, it has its limitations. Firstly, even though we are using growth rate as a predictor of more aggressive disease, this has not been validated. Secondly, images were not centrally reviewed, although a uro-oncologist at each institution did review the masses and recorded the tumour characteristics. Thirdly, linear size (diameter) was used to measure tumour size and growth. Although this is the most clinically utilized and reproducible way to follow masses, it may not be as representative of biologic growth as calculating tumour volume. Finally, other potentially predictive patient and tumour characteristics, such as sex, degree of endophytic component and tumour location, were not available for analysis.

\section{Conclusion}

Based on this large multicentre prospective clinical trial on patients with SRMs on AS, it has been demonstrated that patient characteristics, such as age and symptoms at diagnosis and tumour characteristics, such as consistency and diameter of the mass at diagnosis, are not predictive of tumour growth. These findings complicate the development of evidence-based follow-up schemes for renal masses on AS. Finally, it is clear that further knowledge is needed on the growth kinetics of SRMs, as well as on prognostic factors and factors of disease progression.

Competing interests: Dr. Finelli is Advisory Board member for Amgen, Astellas and Janssen. He has also received honoraria from Amgen, Astellas, Janssen, Paladin and Astra Zeneca. Dr. Finelli has also participated in clinical trials in the past 2 years for Amgen, Astellas, Janssen and Ferring. Dr. Fleshner is a member of the Advisory Board for Amgen, Janssen, Astellas, and Eli Lily and has received honoraria for his roles. He has also participated in clinical trials for Amgen, Janssen, Medivation, the Ontario Institute for Cancer Research, and Prostate Cancer Canada. Dr. Morash does not have an affiliation with a pharmaceutical, medical device or communications organization. Dr. Rendon is a member of the Advisory Board and the Speakers bureau for Amgen, Astellas, Ferring and Janssen. . Dr. Siemens is the Editor-in-chief at CUAJ. Dr. Drachenberg has attended Advisory Boards for Astellas and Janssen and has been a speaker for Amgen and Actavis (formerly Watson). He has also been an investigator in clinical trials run by Cancer Care Manitoba (CCMB). Dr. Chin and Ms. Basiuk have no competing financial or personal interests.

This paper has been peer-reviewed.

\section{References}

1. Siegel R, Naishadham D, Jemal A. Cancer statistics, 2013. CA Cancer J Clin 2013;63:11-30. http:// dx.doi.org/10.3322/caac.21166

2. Kane CJ, Mallin K, Ritchey J, et al. Renal cell cancer stage migration: Analysis of the National Cancer Data Base. Cancer 2008;113:78-83. htrp://dx.doi.org/10.1002/cncr.23518 
3. Hollingsworth JM, Miller DC, Daignault $S$, et al. Rising incidence of small renal masses: A need to reassess treatment effect. J Natl Cancer Inst 2006;98:1331-4. http://dx.doi.org/10.1093/inci/dij362

4. Campbell SC, Novick AC, Belldegrun A, et al. Guideline for management of the clinical T1 renal mass. J Urol 2009;182:1271-9. http://dx.doi.org/10.1016/i.uro.2009.07.004

5. Huang WC, Levey AS, Serio AM, et al. Chronic kidney disease after nephrectomy in patients with renal cortical tumours: A retrospective cohort study. Lancet Oncol 2006;7:735-40. http://dx.doi.org/10.1016/ S1470-2045(06)70803-8

6. Thompson RH, Boorijan SA, Lohse CM, et al. Radical nephrectomy for ptla renal masses may be associated with decreased overall survival compared with partial nephrectomy. J Urol 2008; 179:468-71; discussion 472-3. http://dx.doi.org/10.1016/i.juro.2007.09.077

7. Jewett MAS, Martar K, Basiuk J, et al. Active surveillance of small renal masses: Progression patterns of early stage kidney cancer. Eur Urol 2011;60:39-44. http://dx.doi.org/10.1016/i.eururo.2011.03.030

8. Jemal A, Siegel R, Ward E, et al. Cancer statistics, 2009. CA Cancer J Clin 2009:59:225-49. hitp:// dx.doi.org/10.3322/caac.20006

9. Mason RJ, Abdolell M, Trottier G, et al. Growth kinetics of renal masses: Analysis of a prospective cohort of patients undergoing active surveillance. Eur Urol 2011;59:863-7. http://dx.doi.org/10.1016/i. eururo.2011.02.023

10. Frank I, Blute ML, Cheville IC, et al. Solid renal tumors: An analysis of pathological features related to tumor size. J Urol 2003;170:2217-20. http://dx.doi.org/10.1097/01.ju.0000095475.12515.5e

11. Ljungberg B, Cowan NC, Hanbury DC, et al. EAU guidelines on renal cell carcinoma: the 2010 update. Eur Urol 2010;58:398-406. http://dx.doi.org/10.1016/i.eururo.2010.06.032

12. Hever $R$, Gill IS, Guazzoni $G$, et al. A critical analysis of the actual role of minimally invasive surgery and active surveillance for kidney cancer. Eur Urol 2010;57:223-32. http://dx.doi.org/10.1016/i. eururo.2009.10.023

13. Snyder ME, Bach A, Kattan MW, et al. Incidence of benign lesions for clinically localized renal masses smaller than $7 \mathrm{~cm}$ in radiological diameter: Influence of sex. J Urol 2006; 176:2391-5; discussion 2395-6. http://dx.doi.org/10.1016/i.juro.2006.08.013
14. Dechet CB, Zincke H, Sebo TJ, et al. Prospective analysis of computerized tomography and needle biopsy with permanent sectioning to determine the nature of solid renal masses in adults. J Urol 2003;169:71-4. http://dx.doi.org/10.1016/50022-5347(05)64038-4

15. Chawla SN, Crispen PL, Hanlon AL, et al. The natural history of observed enhancing renal masses: Metaanalysis and review of the world literature. J Urol 2006;175:425-31. http://dx.doi.org/10.1016/ S0022-5347(05)00148-5

16. Crispen PL, Viterbo R, Boorijan SA, et al. Natural history, growth kinetics, and outcomes of untreated clinically localized renal tumors under active surveillance. Cancer 2009;115:2844-52. http://dx.doi. org/10.1002/cncr.24338

17. Bosniak MA. The current radiological approach to renal cysts. Radiology 1986;158:1-10.

18. Abdolell M, LeBlanc M, Stephens D, et al. Binary partitioning for continuous longitudinal data: Categorizing a prognostic variable. Stat Med 2002;21:3395-409. http://dx.doi.org/10.1002/sim.1266

19. Segal MR. Extending the elements of tree-structured regression. Stat Methods Med Res 1995;4:219-36. http://dx.doi.org/10.1177/096228029500400304

20. Thompson RH, Kurta JM, Kaag M, et al. Tumor size is associated with malignant potential in renal cell carcinoma cases. J Urol 2009;181:2033-6. http://dx.doi.org/10.1016/i.juro.2009.01.027

21. Umbreit EC, Shimko MS, Childs MA, et al. Metastatic potential of a renal mass according to original tumour size at presentation. BJU Int 2012;1092:190-4; discussion 194. http://dx.doi.org/10.1111/i.1464410X.2011.10184.X

22. Smaldone MC, Kutikov A, Egleston BL, et al. Small renal masses progressing to metastases under active survellance: A systematic review and pooled analysis. Cancer 2012;118:997-1006. http://dx.doi. org/10.1002/cncr.26369

Correspondence: Dr. Michael Organ, Faculty of Medicine, Department of Urology, Dalhousie University, 1276 South Park St., Room 293, 5 Victoria, Halifax, NS; organ@dal.ca 